\title{
Efficiently Enhancing Low-dielectric Properties and Chemical Resistances of Addition-cure Liquid Silicone Rubber by Filling With Silicone-modified Eucommia Gum Nanofiller
}

\section{Chaohua Li}

Hunan Normal University

Shengpei Su ( $\square$ shengpei_academi@sohu.com )

Hunan Normal University https://orcid.org/0000-0003-4225-1468

\section{Bikui Wang}

Hunan Normal University

Jijia Zhou

Hunan Normal University

\section{Research Article}

Keywords: electronic materials, nanostructures, sol-gel chemistry, dielectric properties, interfaces

Posted Date: March 30th, 2021

DOI: https://doi.org/10.21203/rs.3.rs-335738/v1

License: (c) (1) This work is licensed under a Creative Commons Attribution 4.0 International License.

Read Full License

Version of Record: A version of this preprint was published at Journal of Materials Science: Materials in Electronics on July 9th, 2021. See the published version at https://doi.org/10.1007/s10854-021-06566-2. 


\section{Abstract}

It is of great interest and remains a challenge to simultaneously improve the low-dielectric properties and chemical resistances of addition-cure liquid silicone rubber (ALSR). In this work, we proposed an efficient approach to address this issue by filling silicone-modified eucommia gum nanofiller (SEUG) into ALSR. By adding $5 \mathrm{wt} \%$ SEUG, the dielectric constant of the SEUG/ALSR composite rubber was significantly lower than that of neat ALSR both at $10^{2} \mathrm{~Hz}, 10^{3} \mathrm{~Hz}$ and $10^{4} \mathrm{~Hz}$. Simultaneously, the SEUG/ALSR composite rubber exhibited better mechanical and insulation properties than the neat ALSR after $\mathrm{HCl}$, $\mathrm{NaCl}$, and oil resistance tests. Our findings demonstrated the great potential for fabricating silicone rubber with low-dielectric properties and erosion resistance by the utilization natural biomass rubber material, endowing it with excellent mechanical performance and degradability.

\section{Introduction}

Addition-cure liquid silicone rubber (ALSR) is a packaging material that has been widely application in electronics, electrical power, and electrical fields [1-5] because of its excellent high- and low-temperature resistance, good elastic properties, and lack of by-product residues or volatiles. However, practical application of pure ALSR is severely hindered by its poor mechanical properties $[6,7]$ and poor erosion resistance [8-14]. Packaging material failure due to corrosion often causes great harm to electronic components, which significantly threatens the security of electrical systems. Therefore, it is imperative to develop an effective strategy to enhance the mechanical properties and erosion resistance of ALSR. To overcome these problems, an effective method is to disperse nanoparticles into the ALSR, such as clay [15], $\mathrm{TiO}_{2}$ [16-19], $\mathrm{SiO}_{2}$ [20-23], graphene [24-27], carbon nanotubes [28,29], boron nitride (BN) [30,31], and aluminum nitride (AIN) [32]. However, due to the high permittivities and poor compatibility of these materials with ALSR, the dielectric constant of the ALSR significantly increases [33]. However, ALSR is widely used as an insulating packing material for electronic devices, such as central processing units and integrated circuit, and thus, a low dielectric constant and loss factor are required [34,35]. A low dielectric constant helps to decrease the time of signal propagation, while a low loss factor aids in decreasing the heat production. Such disadvantages limit the further applications of ALSR. Therefore, it is necessary to develop a new method to modify the ALSR for the improvement of the low dielectric constant and erosion resistance. Nevertheless, very few studies on ALSR have addressed this issue.

Eucommia ulmoides gum (EUG) is a special natural rubber obtained from eucommia ulmoides Oliv, which grows mainly in China. EUG exhibits many excellent properties due to its molecular structure containing long-chain trans-1,4-polyisoprene, such as strong insulation properties, a low dielectric constant, water resistance, chemical resistance, and a high toughness. Furthermore, the development of EUG is listed in the national key research program of China because EUG is renewable and degradable. Nevertheless, some drawbacks of pure EUG, such as its poor high-temperature resistance and elastic properties, limit its widespread application. Instead, EUG shows good corrosion protection properties as the nanofiller in composite materials and has shown great potential for application in anticorrosion materials. For example, Bo Chen et al. [36] added an epoxidized biomass eucommia gum into an epoxy 
composite coating to obtain excellent anticorrosive performance. To the best of our knowledge, there have been few studies on EUG fillers in silicone rubber.

In this work, we selected silicone-modified eucommia gum nanofiller as the filler to systematically study its effects on the mechanical, insulation, dielectric, and chemical-resistance properties of ALSR. Through this study, we expect to contribute to the preparation of ALSR with extraordinary dielectric properties and chemical resistance. This work also provides a new and feasible strategy for manufacturing degradable ALSR.

\section{Experimental}

\subsection{Materials}

Eucommia ulmoides gum (EUG) was purchased from Xiangxi Laojiao Biological Co., Ltd. (China). Xylene and hydrogen peroxide (30\% wt) were purchased from China National Pharmaceutical Group Co. The platinum catalyst (platinum content was 5000 ppm) and 1-ethynylcyclohexanol were supplied by ShinEtsu Co., Ltd., Japan. Poly(hydromethylsiloxane) -1(PHMS-1, 0.1 wt\% hydride), Poly(hydromethylsiloxane)-2 (PHMS-2, $0.5 \mathrm{wt} \%$ hydride), and vinyl-terminated poly(dimethylsiloxane) (VPDMS, 0.28 wt\% vinyl) were obtained from Elkem Silicones Co., Ltd., China.

\subsection{Synthesis of silicone-modified eucommia gum nanofiller (SEUG)}

Silicone-modified eucommia gum nanofiller (SEUG) was synthesized through our developed two-step hydrosilylation sol-gel reaction, which is shown in Fig. 1. In step one, the eucommia ulmoides was purified. The eucommia ulmoides shell $(150 \mathrm{~g})$ was cut into small particles, which were then transferred to a four-necked $5000-\mathrm{mL}$ round-bottom flask equipped with a magnetic stirrer, and xylene $(3500 \mathrm{~g})$ was added. After this, the mixer was heated to $80^{\circ} \mathrm{C}$, and a mixture of $\mathrm{H}_{2} \mathrm{O}_{2}(10 \mathrm{~g})$ and pure water $(150 \mathrm{~g})$ was added dropwise under stirring. The mixture was stirred for $6 \mathrm{~h}$ at $80^{\circ} \mathrm{C}$. Eucommia ulmoides $(3538 \mathrm{~g})$ was obtained after centrifugation to remove the impurities and water.

In step two, the hydrosilylation reaction occurred. The eucommia ulmoides after purification $(3538 \mathrm{~g})$ and the platinum catalyst $(0.5 \mathrm{~g})$ were added to a $5000-\mathrm{mL}$ four-neck round bottom flask. The mixture was first stirred for $0.5 \mathrm{~h}$ at $60^{\circ} \mathrm{C}$. Poly (hydromethylsiloxane) (PHMS-1 100g) was added dropwise to the flask. The mixture was stirred for $3 \mathrm{~h}$ at $60^{\circ} \mathrm{C}$ and then heated to $90^{\circ} \mathrm{C}$ for $3 \mathrm{~h}$. Reduced-pressure distillation was appl

\subsection{Preparation of the SEUG/ALSR composite rubbers}

VPDMS (100 g) and SEUG $(5,10$, or $15 \mathrm{~g})$ were uniformly mixed in a high-speed disperser to obtain the nano-dispersion system. A suitable quantity of PHMS-2, 1-ethynylcyclohexanol, and Karstedt's catalyst was vigorously stirred using an electric mixer. The mixture was subsequently vulcanized at $120^{\circ} \mathrm{C}$ for $1 \mathrm{~h}$, after which the ALSR samples were successfully prepared. The compositions of the ALSR samples are 
listed in Table 1. The SEUG was uniformly dispersed in the ALSR matrix.ied to remove the xylene, and SEUG(135) was obtained. The synthesis scheme is shown in scheme. 1.

\subsection{Characterization}

\subsubsection{Fourier-transform infrared spectroscopy}

The liquid sample was first coated on the surface of the $\mathrm{KBr}$ slice and was then analyzed by Fouriertransform infrared spectroscopy (FT-IR, Tensor 27, Bruker Optics, Germany) from 4000 to $400 \mathrm{~cm}-1$ with a resolution of $4 \mathrm{~cm}-1$ and 16 total scans.

\subsubsection{Surface morphology test}

The fractured cross sections of the nanocomposites were characterized by scanning electron microscopy (SEM, Hitanchi S-4700).

\subsubsection{Mechanical properties test}

The tensile strength and elongation at break of the ALSR specimens were measured using a universal testing machine (HD-WDW-100A, Xiamen HaiDa Inc., China) according to ISO 37-2005. The Shore A hardness of the samples was conducted with a Shore A durometer (LX-A, Shanghai Liuling Instruments Factory, China) in accordance with ISO 7619-1-2004.

\subsubsection{Insulation performance test}

The volume resistivities of the ALSR specimens were measured using a surface volume resistivity testing machine (ZST-121, ZhongHangShiDaixin Instrument Equipment Inc., China) according to ASTM D 257.

\subsubsection{Dielectric performance test}

The dielectric constant of the SEUG/ALSR composite rubber specimens were measured using a dielectric analyzer DEA-2970 coupled with a temperature controller 2000 (TA Instruments Inc., USA) using the compression-molded specimens with thicknesses of $0.3-0.6 \mathrm{~mm}$. The measurements were performed at a frequency of $10^{2} \mathrm{~Hz}, 10^{3} \mathrm{~Hz}$ and $10^{4} \mathrm{~Hz}$.

\subsubsection{Chemical resistance test}

The chemical resistance of the ALSR was tested according to ISO 1817-2005. The effects of liquids (acid $(\mathrm{HCl})$, base $(\mathrm{NaOH})$, salt $(\mathrm{NaCl})$, and aviation oil) on different ALSR samples were studied. In each case, five pre-weighed samples were dipped in the respective chemical reagents for $30 \mathrm{~d}$. They were then removed, immediately washed with distilled water, and dried by pressing both sides of the samples with filter paper at room temperature. The mechanical properties and insulation properties of the ALSR samples were measured. The variation of the performance were calculated. 


\section{Results And Discussion}

\subsection{Characterization of SEUG}

To address the issue of poor compatibility, the SEUG was synthesized through a hydrosilylation reaction, which is shown in Fig. 1. Fig. 2 shows the FT-IR spectra of the SEUG and EUG from which the functional groups were identified. The characteristic peaks at 1460 and $1380 \mathrm{~cm}-1$ were attributed to the bending vibrations of the methyl groups, which indicated that EUG and SEUG both contained methyl groups. The peak at $1665 \mathrm{~cm}-1$ was attributed to $C=C$ stretching vibrations. The characteristic peak at $2869 \mathrm{~cm}-1$ was assigned to the stretching vibrations of the $\mathrm{CH}$ in $\mathrm{R} 2 \mathrm{C}=\mathrm{CHR}$. The relative intensity of the $\mathrm{C}=\mathrm{C}$ peak for the SEUG was significantly weakened compared to that of the EUG, suggesting that the $\mathrm{C}=\mathrm{C}$ participated in the hydrosilylation reaction. However, the characteristic peak was still present, which indicated that $\mathrm{C}=\mathrm{C}$ was present in the SEUG. Additionally, as for the SEUG, one new peak appeared at $2128 \mathrm{~cm}-1$, which was attributed to $\mathrm{Si}-\mathrm{H}$. The peaks at 1099 and $1024 \mathrm{~cm}-1$ were characteristic of Si-O-Si groups. Thus, the silanization of EUG occurred through the hydrosilylation reaction.

\subsection{Surface morphologies of SEUG/ALSR composite rubbers}

To further confirm that SEUG was dispersed uniformly in the ALSR, observation of the morphologies of the SEUG/ALSR composite rubbers and neat ALSR were examined using SEM. The surface of the neat ALSR was smooth (Fig. 3, ALSR-1). Although uniform plications were evident on the surfaces of the composite rubbers containing $5 \mathrm{wt} \%$ SEUG (Fig. 3, ALSR-2) Ønone evident independent particles was showed. This was evident where the dispersion of the SEUG nanofiller was sparse in the ALSR-2, which verified that most of the SEUG nanofiller was encapsulated in the ALSR.

The uniformity of the plications also indicated that the compatibility of the ALSR with the SEUG was significantly enhanced.However, when the doped contents of the SEUG further increased to $10 \mathrm{wt} \%$, the surface of the SEUG/ALSR composite rubber (Fig. 3, ALSR-3) showed showed some evident particles compared to ALSR-2. The small particles were free macromolecular SEUG, and they could aggregate to form large particles. As shown in Fig. 3 (ALSR-4), upon further increasing the SEUG content to $15 \mathrm{wt} \%$, the number of small particles was reduced, while the volumes of the large particles were larger than those of the ALSR-3. This verified that more particle aggregation occurred.

\subsection{Mechanical properties of SEUG/ALSR composite rubbers}

To understand the effect of the SEUG on the mechanical properties of the composite rubbers, the tensile strength, elongation at break, and Shore A hardness were determined. The tensile strength of the SEUG/ALSR composites as a function of the mass fraction of SEUG is shown in Fig. 4. In the case of the neat ALSR, the tensile strength value was about 1.35 MPa. For the composite rubbers with different doped contents of SEUG, the tensile strength increased first as the doped content of SEUG increased. The maximum reached $1.71 \mathrm{MPa}$ when the content of SEUG was $5 \%$. This can be explained as follows. On the one hand, SEUG had a larger relative molecular mass and longer molecular chains. Thus, it possessed 
a higher mechanical strength than the ALSR, which led to increased tensile strengths of the SEUG/ALSR composite rubbers. On the other hand, the Si-H groups in the SEUG could react with the VPDMS through an addition reaction during the curing process, resulting in the formation of a closer interpenetrating network structure. The promotion of the tensile strength was the direct result of the richer crosslinking densities. However, the tensile strength decreased abruptly to $1.21 \mathrm{MPa}$ after increasing the content of the SEUG nanoplate filler to $10 \mathrm{wt} \%$. This indicated that with further addition of SEUG, the free SEUG could further form large particles due to agglomeration. The miscibility and compatibility of the SEUG with the ALSR could be reduced, thereby leading to a decreased mechanical strength of the SEUG/ALSR composite rubbers, which was confirmed by compatibility tests, as shown in Fig. 3.

The elongation at break and Shore A hardness values of the SEUG/ALSR composite rubbers are shown in Figs. 4 and 5, respectively. As the content of the SEUG nanoplate filler increased, the Shore hardness steadily decreased from 32 to 18 . In contrast, the elongation at break of the ALSR strongly increased with the SEUR content. This indicated that the toughening of the SEUG/ALSR composite rubbers increased compared to that of the neat ALSR. This agreed with recently reported results $[37,38]$.

\subsection{Dielectric performance of SEUG/ALSR composite rubbers}

The dielectric constants $(\varepsilon r)$ of the pure ALSR and SEUG/ALSR composites were measured at frequency of $10^{2} \mathrm{~Hz}, 10^{3} \mathrm{~Hz}$ and $10^{4} \mathrm{~Hz}$ at room temperature, and the results are shown in Fig. 6 . As the SEUG concentration increased, the dielectric constant first decreased and then increased. The possible mechanism through which the SEUG affected the low-dielectric properties of the ALSR is demonstrated in Fig. 7. Based on previous research, the relative dielectric constant of the filled ALSR was found to be governed by the mobility of the PDMS chains, the inherent permittivity of the nanofiller, and the polarization associated with the silicone and particles $[39,40]$. It is well-known that the EUG had similar permittivities (2-3.2) to that of pure ALSR (2-3). Therefore, the influence of the SEUG nanofiller's inherent permittivity on the overall permittivities of the composites was negligible. As for the composite rubber with a SEUG doping content of $5 \mathrm{wt} \%$ (ALSR-2), the dielectric constant was lower than that of the neat ALSR both at $10^{2} \mathrm{~Hz}, 10^{3} \mathrm{~Hz}$ and $10^{4} \mathrm{~Hz}$. This suggested that the introduction of SEUG enhanced the low dielectric properties of the composite rubber compared to those of the neat ALSR. This phenomenon can be explained as follows. On the one hand, the SEUG/ALSR composite exhibited good dispersion of the SEUG particles. The SEUG particles were highly encapsulated by the ALSR matrix (Fig. 3, ALSR-2). No distinct interfacial polarization between the SEUG and ALSR phases was evident because of the hydrosilylation reaction during the surface modification of the SEUG nanoparticles. On the other hand, the $\mathrm{Si}-\mathrm{H}$ groups in the SEUG could react with the VPDMS through an addition reaction during the curing process, resulting in the formation of closer interpenetrating network structures, which restricted the mobility of the polysiloxane chains in the composite rubbers. This restriction inhibited the electrical polarization because the material lost its freedom to relax under an applied voltage, and thus, the dielectric constant decreased. However, as the concentration of SEUG further increased, the dielectric constant of the composite rubbers exhibited a notable rise with increasing SEUG content, which was ascribed to the interfacial polarization (Maxwell-Wagner polarization) at the interface between the ALSR 
or SEUG. As shown in Fig. 8, the small SEUG particles were created by the aggregation of free macromolecular SEUG, and the small SEUG particles further aggregated to form large SEUG particles. The particle agglomeration was confirmed by the SEM analysis. The presence of more interfacial polarization at the interface between the ALSR and SEUG particles resulted in a higher permittivity as the SEUG concentration increased.

\subsection{Chemical resistance properties of SEUG/ALSR composite rubbers}

To understand the effect of the SEUG on the chemical resistance properties of the composite rubbers, the tensile strength (mechanical properties) and volume resistivity (insulation performance) were measured after different chemical resistance tests. The effect of the SEUG on the chemical resistance properties of the SEUG/ALSR composite rubbers could be observed by the performance of tensile strength and volume resistivity. The performance after the $\mathrm{HCl}, \mathrm{NaCl}$, and oil resistance tests with different SEUG contents is shown in Figs. 8, 9, and 10, respectively.

It could be seen that the tensile strength and volume resistivity of the SEUG/ALSR composite rubbers after the $\mathrm{HCl}, \mathrm{NaCl}$, and oil resistance tests were similar. The tensile strength and volume resistivity initially increased with the SEUR content, which confirmed the enhancements of the chemical $(\mathrm{HCl}, \mathrm{NaCl}$, and oil) resistance properties, and then reached a maximum value at $5 \mathrm{wt} \%$. When the SEUR content increased further to $10 \mathrm{wt} \%$, a gradual decrease occurred, which indicated that the chemical resistance properties degraded.However, the variation of the performance after the $\mathrm{NaOH}$ resistance test with different SEUG contents is shown in Fig. 11. The tensile strength and volume resistivity decreased with the SEUR content, indicating that the $\mathrm{NaOH}$ resistance properties degraded significantly as the SEUR content was increased.

\subsection{Reasonable chemical resistance promotion mechanism}

Based on the results presented above, it was reasonably concluded that the $\mathrm{HCl}, \mathrm{NaCl}$, and oil resistance properties of the SEUG/ALSR composite rubbers with $5 \mathrm{wt} \%$ SEUG were remarkably enhanced compared to those of the neat ALSR. A possible mechanism through which the SEUG enhanced the $\mathrm{HCl}, \mathrm{NaCl}$, and oil resistances of the SEUG/ALSR composite rubbers is demonstrated in Fig. 12. The SEUG has better chemical resistance properties than ordinary ALSR due to the larger relative molecular mass, the long molecular chains, and the excellent molecular regularity. Therefore, the $\mathrm{HCl}, \mathrm{NaCl}$, and oil resistance properties of the SEUG/ALSR composite rubbers were enhanced significantly with the introduction of SEUG. Additionally, the SEUG had active Si-H groups that could react with the VPDMS during the curing process, not only resulting in the formation of closer three-dimensional cross-linked structures but also causing good dispersion in the composite rubber. The much greater crosslinking densities acted as better physical barriers to prevent the electrolytes from entering the ALSR. Based on these two factors, the chemical resistance properties initially increased with the SEUR content and reached a maximum value at $5 \mathrm{wt} \%$. However, when the doped SEUG content was increased to $10 \mathrm{wt} \%$ in the composite rubbers, free SEUG was present (Fig. 3). The free SEUG could further form large particles due to the agglomeration, leading to an increase in the molecular chain gap and worse dispersion in the composite rubber, which 
were both beneficial for the formation of diffusion channels for electrolytes. This could promote the entry of the corrosive medium into the ALSR, as illustrated in Fig. 12. Thus, these factors can explain the degradation of the chemical resistance properties for the SEUG/ALSR composite rubbers with $10 \mathrm{wt} \%$ SEUG.

The above analysis showed that SEUG significantly reduced the $\mathrm{NaOH}$ resistances of the SEUG/ALSR composite rubbers. We proposed a possible mechanism for this, as shown in Fig. 14. For the EUG, when extracted by the organic solvent extraction method, some bio-based macromolecules remained in the EUG, such as chlorogenic acid and geniposide crystals (the structures are shown in Fig. 13). As the reaction proceeded, these bio-based macromolecules were injected into the macromolecular chains of the SEUG/ALSR composite rubbers, as shown in Fig. 15. The SEUG/ALSR composite rubber chains would be destroyed by the reaction of sodium hydroxide with these bio-based macromolecules when the samples were immersed in the sodium hydroxide. Thus, the $\mathrm{NaOH}$ resistance of the ALSR was reduced. Furthermore, this could provide a new direction for the degradation of SEUG/ALSR composite rubbers.

\section{Conclusions}

In summary, ALSR, featuring outstanding low-dielectric properties and chemical resistance, was successfully prepared by filling it with a silicone-modified eucommia gum nano-filler. Silicone-modified eucommia gum (SEUG) was successfully prepared through a hydrosilylation reaction. As a nanofiller, the SEUG/ALSR composite rubber with SEUG contents up to $5 \mathrm{wt} \%$ could be easily processed. The Si-H group in the SEUG could react with VPDMS, not only resulting in good compatibility with the ALSR and preventing the SEUG from agglomeration but also leading to the formation of the much richer crosslinking densities, which was favorable for increasing the tensile strength and toughening of the ALSR. In addition, the dielectric constant of the SEUG/ALSR composite rubber were lower than 1.84, 1.79 and 1.75 at $10^{2} \mathrm{~Hz}, 10^{3} \mathrm{~Hz}$ and $10^{4} \mathrm{~Hz}$, respectively, which would be beneficial for decreasing the time of signal propagation in electronic devices and the long-term operation of capacitors owing to the low heat dissipation. Furthermore, because of the richer crosslinking densities and the relatively larger molecular chains, the SEUG/ALSR composite rubber containing $5 \mathrm{wt} \%$ SEUG showed significantly enhanced $\mathrm{HCl}$, $\mathrm{NaCl}$, and oil resistance performances. After $30 \mathrm{~d}$ of immersion in solution, the mechanical and insulation properties of the SEUG/ALSR composite rubber improved significantly compared to those of the neat ALSR. Moreover, the $\mathrm{NaOH}$ resistance of the ALSR was reduced by the bio-based macromolecules of the SEUG, which may provide a new direction for the degradation of SEUG/ALSR composite rubbers.

\section{Declarations}

Acknowledgments

This research did not receive any specific grant from funding agencies in the public, commercial, or notfor-profit sectors. 


\section{References}

1. K. , S. Sreekantan, M. Devarajan, K. Mohamed, Effect of hybrid filler ratio and filler particle size on thermal conductivity and oil bleed of polydimethylsiloxane/Al203/ZnO liquid thermal filler for microelectronics packaging applications. Journal of Materials Science: Materials in Electronics 2021;32(11):861-874 https://link.springer.com/article/10.1007/s10854-020-04864-9

2. Weizhen Fang, Xingrong Zeng, Xuejun Lai, Hongqiang Li, Wanjuan Chen,Yajun Zhang.Thermal degradation mechanism of addition-cure liquid silicone rubber with urea-containing silane. Thermochimica Acta 2015;605(4):2836 https://www.sciencedirect.com/science/article/abs/pii/S004060311500060X

3. Chixin Xie, Xuejun Lai, Hongqiang Li, Xingrong Zeng.Effective improvement of anti-tracking of addition-cure liquid silicone rubber via charge dissipation of fluorosilane-grafted silica.Polymer Degradation and Stability 2019;167(9):250258 https://www.sciencedirect.com/science/article/pii/S0141391019302174

4. Liu, C. Ma, Y. Li, Z. Yin, J. Su , J. Gao. A study on the effect of cross-linkers on pervaporation performance of room temperature vulcanised silicone rubber membranes for butanol recovery. Plastics, Rubber and Composites 2018; 47(5):202207 https://doi.org/10.1080/14658011.2018.1468586

5. Hongqiang Li, Yanping Li, Tongyi Wu, Xiaofeng Liao, TianLiu, Xuejun Lai, Xingrong Zeng.Plasma resistance of addition-cure liquid silicone rubber with Ureido-attached MQ silicone resin.Surfaces and Interfaces 2019;14(3):5560 https://www.sciencedirect.com/science/article/pii/S2468023018303201

6. Lizhen Guo, Xinxin Yang, Fuhao Dong, Yuehan Qian, Jiawen Guo, Xiangyu Lin, Hiba Shaghaleh, Weiqi Liu, Xu Xu, Shifa Wang, Shiwei Liu.A pinene-based silane crosslinker for improved mechanical strength/transparency of room-temperature vulcanizing silicone rubber.Materials Chemistry and Physics 2020;247(6):122868 https://www.sciencedirect.com/science/article/abs/pii/S0254058420302479

7. Yunfei S.L, Chongguang Zang, Yulong Zhang.Effect of the structure of hydrogen-containing silicone oil on the properties of silicone rubber.Materials Chemistry and Physics 2020;10(3):122734 https://www.sciencedirect.com/science/article/abs/pii/S0254058420301139

8. Yanping Li, Xingrong Zeng, Xuejun Lai, Hongqiang Li, Weizhen Fang.Effect of the platinum catalyst content on the tracking and erosion resistance of addition-cure liquid silicone rubber.Polymer Testing 2017;63(10):92-100 https://www.sciencedirect.com/science/article/abs/pii/S014294181730658X

9. Weizhen Fang, Xuejun Lai, Hongqiang Li, Wanjuan Chen, Xingrong Zeng, Liping Zhang, Siguang Yang.Effect of urea-containing anti-tracking additive on the tracking and erosion resistance of addition-cure liquid silicone rubber.Polymer Testing 2014;37(8):1927 https://www.sciencedirect.com/science/article/abs/pii/S0142941814000932

10. Yajun Zhang, Xingrong Zeng, Xuejun Lai, Hongqiang Li, Xiaoyi Huang.Significant improvement of urethane-containing silane on the tracking and erosion resistance of silicone rubber/silica 
nanocomposite by enhancing the interfacial effect.Polymer Testing 2018;69(8):16-

25 https://www.sciencedirect.com/science/article/abs/pii/S0142941818305464

11. Jiedong Qiu, Xuejun Lai, Weizhen Fang, Hongqiang Li, Xingrong Zeng.An efficient strategy for simultaneously improving tracking resistance and flame retardancy of addition-cure liquid silicone rubber.Polymer Degradation and Stability 2017;144(10):176-

186 https://www.sciencedirect.com/science/article/pii/S0141391017302379

12. Chixin Xie, Xingrong Zeng, Weizhen Fang, Xuejun Lai, Hongqiang Li.Effect of alkyl-disubstituted ureido silanes with different alkyl chain structures on tracking resistance property of addition-cure liquid silicone rubber.Polymer Degradation and Stability 2017;142(8):263-

272 https://www.sciencedirect.com/science/article/pii/S0141391017302057

13. Tian Liu, Xingrong Zeng, Weizhen Fang, Xuejun Lai, Hongqiang Li.Synthesis of a novel hydantoincontaining silane and its effect on the tracking and bacteria resistance of addition-cure liquid silicone rubber.Applied Surface Science 2017;423(11):630640 https://www.sciencedirect.com/science/article/abs/pii/S0169433217317737

14. Tongyi Wu, Xuejun Lai, Fengjiao Liu, Hongqiang Li, Xingrong Zeng.Efficiently enhancing the tracking and erosion resistance of silicone rubber by the synergism of fluorine-containing polyphenylsilsesquioxane and ureido-containing MQ silicone resin.Applied Surface Science 2018;459(11):483-491 https://www.sciencedirect.com/science/article/abs/pii/S0169433218321408

15. Maryam Sarkarat, Michael Lanagan, Dipankar Ghosh, Andrew Lottes, Kent Budd, Ramakrishnan Rajagopalan.High field dielectric properties of clay filled silicone rubber composites.Materials Today Communications 2020;23(6):100947 https://www.sciencedirect.com/science/article/pii/S2352492820301008

16. Liyun Yu , A. L. Skov. Silicone rubbers for dielectric elastomers with improved dielectric and mechanical properties as a result of substituting silica with titanium dioxide. International Journal of Smart and Nano Materials 2015;9:268-289, https://doi.org/10.1080/19475411.2015.1119216

17. T.Vaimakis-Tsogkas,D.G. Bekas,T.Giannakopoulou,N.Todorova,A.S.Paipetis,N.M.Barkoula.Effect of TiO2 addition/coating on the performance of polydimethylsiloxane-based silicone elastomers for outdoor applications.Materials Chemistry and Physics 2019;223(2):366373 https://www.sciencedirect.com/science/article/abs/pii/S0254058418309696

18. Rezaeian, P. Zahedi, M. S. Loghmani, M. Shahzamani. Comparison between mechanical properties of aged and unaged silicone rubbers filled with titanium dioxide, quartz, aluminium silicate and vulkasil (stype). Plastics, Rubber and Composites 2009;38(7):257-

263 https://doi.org/10.1179/174328909X435401

19. Sohrab Azizi, Gelareh Momen, Claudiane Ouellet-Plamondon, Eric David.Performance improvement of EPDM and EPDM/Silicone rubber composites using modified fumed silica, titanium dioxide and graphene additives.Polymer Testing 2020;84(4):106281 https://www.sciencedirect.com/science/article/abs/pii/S0142941819314497 
20. Dong Liu, Lixian Song, Hongtao Song, Jie Chen, Qiang Tian, Liang Chen, Liangwei Sun, Ai Lu, Chao qiang Huang, Guangai Sun. Correlation between mechanical properties and microscopic structures of an optimized silica fraction in silicone rubber.Composites Science and Technology 2018;165(9):373-379 https://www.sciencedirect.com/science/article/abs/pii/S0266353818307474

21. Dongzhi Chen, Yan Liu, Chi Huang.Synergistic effect between POSS and fumed silica on thermal stabilities and mechanical properties of room temperature vulcanized (RTV) silicone rubbers.Polymer Degradation and Stability 2012;97(3):308-

315 https://www.sciencedirect.com/science/article/pii/S0141391011004150

22. Indumathy, B. Kothandaraman. Effect of a hydroxyl terminated poly dimethyl siloxane, in combination with TESPT, on the mechanical and dynamic properties of silica filled rubber in addition to reducing ethanol emission in combination with TESPT. Plastics, Rubber and Composites 2020;49(1):150-163 https://doi.org/10.1080/14658011.2020.1718325

23. Chiulan, D.M.Panaitescu, E.Radu, A.N.Frone, R.A.Gabor, C.A.Nicolae, G.Jinescu, V.Tofan, ChingaCarrasco. Comprehensive characterization of silica-modified silicon rubbers.Journal of the Mechanical Behavior of Biomedical Materials 2020;101(1):103427 https://www.sciencedirect.com/science/article/pii/S1751616119306149

24. Jianwei Bai, Xia Liao, Erbo Huang, Yong Luo, Qi Yang, Guangxian Lia.Control of the cell structure of microcellular silicone rubber/nanographite foam for enhanced mechanical performance.Materials \& Design 2017;133(11):288298 https://www.sciencedirect.com/science/article/abs/pii/S0264127517307402

25. Cheng Chen, Yan He, Changqing Liu, Huaqing Xie, Wei Yu. Comprehensive excellent performance for silicone-based thermal interface materials through the synergistic effect between graphene and spherical alumina.Journal of Materials Science: Materials in Electronics 2020;31(2):4642-4649 https://link.springer.com/article/10.1007/s10854-020-03016-3

26. Jing Feng, Zhanjun Liu, Dongqing Zhang, ZhaoHe, Zechao Tao, Quangui Guo.Phase change materials coated with modified graphene-oxide as fillers for silicone rubber used in thermal interface applications.New Carbon Materials 2019;34(4):188195 https://www.sciencedirect.com/science/article/pii/S1872580519600119

27. Gui Wang, Xia Liao, Jianming Yang, Wanyu Tang, Yuan Zhang, Qiuyue Jiang, Guangxian Li.Frequency-selective and tunable electromagnetic shielding effectiveness via the sandwich structure of silicone rubber/graphene composite.Composites Science and Technology 2019;184(11):107847 https://www.sciencedirect.com/science/article/abs/pii/S0266353819317932

28. Mengru Chang, Yinglin Li, Lei Xu, Wei Wang, Chunhong Wang, Rui Wang. A novel assembled carbon black/carbon nanotubes (CB/MWCNT) nano-structured composite for pressure-sensitive conductive silicon rubber (SR). Journal of Materials Science: Materials in Electronics 2018;29(11): 2716-2724 https://link.springer.com/article/10.1007/s10854-017-8198-2

29. Pan Song, Jianan Song, Yong Zhang.Stretchable conductor based on carbon nanotube/carbon black silicone rubber nanocomposites with highly mechanical, electrical properties and strain sensitivity. 
Composites Part B: Engineering

2020;3:107979 https://www.sciencedirect.com/science/article/abs/pii/S1359836820300470

30. Pingyuan Liu, Licheng Li, Liming Wang, Ting Huang, Yingbang Yao, Wenrong Xu.Effects of 2D boron nitride (BN) nanoplates filler on the thermal, electrical, mechanical and dielectric properties of high temperature vulcanized silicone rubber for composite insulators. Journal of Alloys and Compounds 2019;774(2):396-404 https://www.sciencedirect.com/science/article/abs/pii/S0925838818336661

31. Qingguo Chen, Banggen Xi, Jinfeng Zhang, Hongda Yang, Xinyu Wang, Minghe Chi.Dielectric properties and thermal conductivity of micro-BN-modified LSR used for high-voltage direct current cable accessories. Journal of Materials Science: Materials in Electronics 2020;31(8):1658316591 https://link.springer.com/article/10.1007/s10854-020-04213-w

32. K. Namitha, S. Ananthakumar, M. T. Sebastian.Aluminum nitride filled flexible silicone rubber composites for microwave substrate applications. Journal of Materials Science: Materials in Electronics 2014;26(11):891-897 https://link.springer.com/article/10.1007/s10854-014-2479-9

33. Boccalero, Claire Jean-Mistral, Maila Castellano, Corrado Boragno.Soft, hyper-elastic and highlystable silicone-organo-clay dielectric elastomer for energy harvesting and actuation applications. Composites Part B: Engineering 2018;146(8):13-

19 https://www.sciencedirect.com/science/article/abs/pii/S1359836818301616

34. Cheng Li, Hong Fan, Tariq Aziz, Carla Bittencourt, Linbo Wu, Deyi Wang, Philippe Dubois.Biobased Epoxy Resin with Low Electrical Permissivity and Flame Retardancy: From Environmental Friendly High-Throughput Synthesis to Properties. ACS Sustainable Chem. Eng 2018;5:8856-

8867 https://pubs.acs.org/doi/10.1021/acssuschemeng.8b01212

35. Yilung Cheng, Chihyen Lee, Weijie Hung, Giinshan Chen, Janshiung Fang.Electrical and reliability characteristics of dielectric stack with low dielectric constant SiCOH and capping SiCNH films.Surface and Coatings Technology 2018;350(9):5763 https://www.sciencedirect.com/science/article/abs/pii/S0257897218306492

36. Bo Chen, Qian Wu, Ji Li, Kaidong Lin, Dongchu Chen, Chengliang Zhou, Tao Wu, Xiaohu Luo, Yali Liu.A novel and green method to synthesize a epoxidized biomass eucommia gum as the nanofiller in the epoxy composite coating with excellent anticorrosive performance. Chemical Engineering Journal 2020;379(1):122323 https://www.sciencedirect.com/science/article/pii/S1385894719317267

37. U. Kang, J.Y. Kim, J. Kim, S.S. Lee, M. Park, S. Lim, C.R. Choe, Effffect of molecular weight between crosslinks on the fracture behavior of rubber-toughened epoxy adhesives. J. Appl. Polym. Sci 2001;79 : 38-48. https://onlinelibrary.wiley.com/doi/10.1002/1097-

4628\%2820010103\%2979\%3A1<38\%3A\%3AAID-APP50>3.0.CO\%3 B2-O

38. J. Rohde, K.M. Le, R. Krishnamoorti, M.L. Robertson, Thermoset blends of an epoxy resin and polydicyclopentadiene. Macromolecules 2016; 49:89608970. https://pubs.acs.org/doi/10.1021/acs.macromol.6b01649 
39. Dan Yang, Yufeng Ni , Xinxin Kong , Dahai Gao, Yan Wang, Tingting Hu , Liqun Zhang,Musselinspired modification of boron nitride for natural rubber composites with high thermal conductivity and low dielectric constant.Composites Science and Technology 2019;177:1825.

https://www.sciencedirect.com/science/article/abs/pii/S0266353818331683

40. Yawen Huang, Xiaonan Wei, Lili Liu, Hongtao Yu, Junxiao Yang, A Novel Pore-Free Strategy via Interfacial Effects in Nanocomposites to Produce Polyethylene with Ultra-Low Dielectric Constants.Materials Letters 2018;232(12):86-

91. https://www.sciencedirect.com/science/article/abs/pii/S0167577X18312862

\section{Tables}

Table 1. Compositions of the SEUG/ALSR composite rubbers.

\begin{tabular}{|lllll|}
\hline Component & ALSR-1 & ALSR-2 & ALSR-3 & ALSR-4 \\
\hline VPDMS & 100 & 100 & 100 & 100 \\
\hline PHMS-2 & 3.5 & 3.5 & 3.5 & 3.5 \\
\hline SEUG & 0 & 5 & 10 & 15 \\
\hline 1-ethynylcyclohexanol & 0.1 & 0.1 & 0.1 & 0.1 \\
\hline Karsted catalyst & 0.1 & 0.1 & 0.1 & 0.1 \\
\hline
\end{tabular}

\section{Figures}




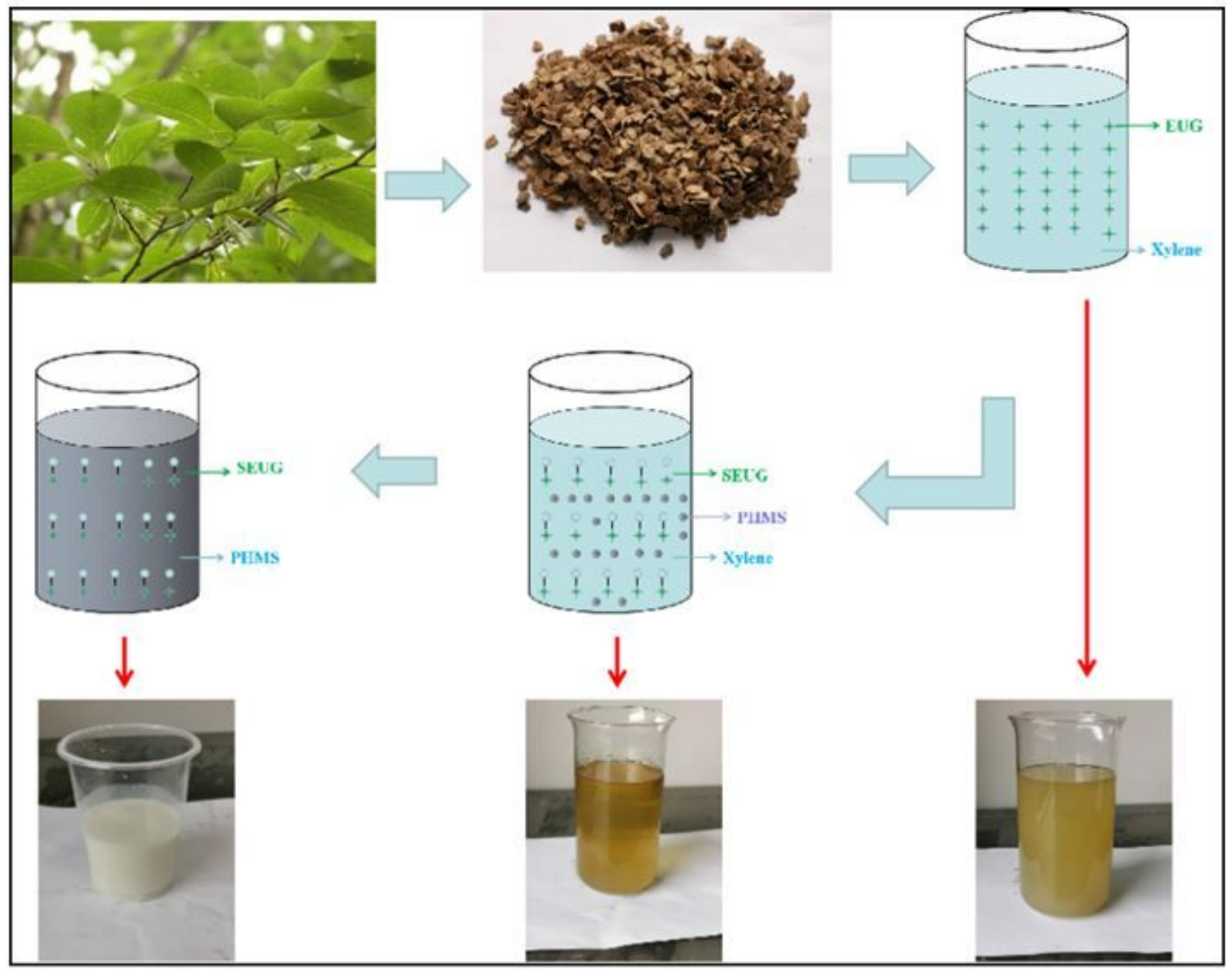

Figure 1

Schematic representation of the synthesis of silicone-modified eucommia gum nanofiller》SEUG囚. 


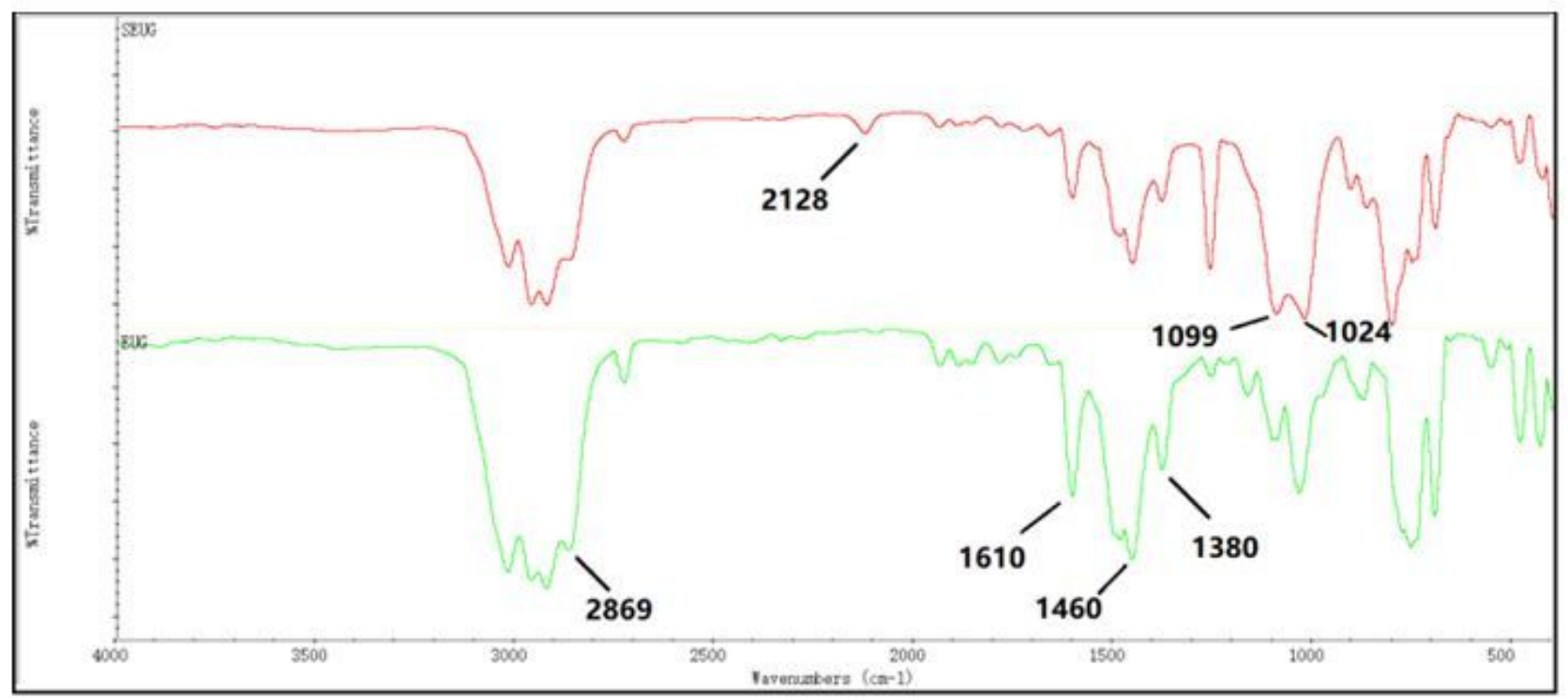

Figure 2

FT-IR spectra of SEUG and EUG. 

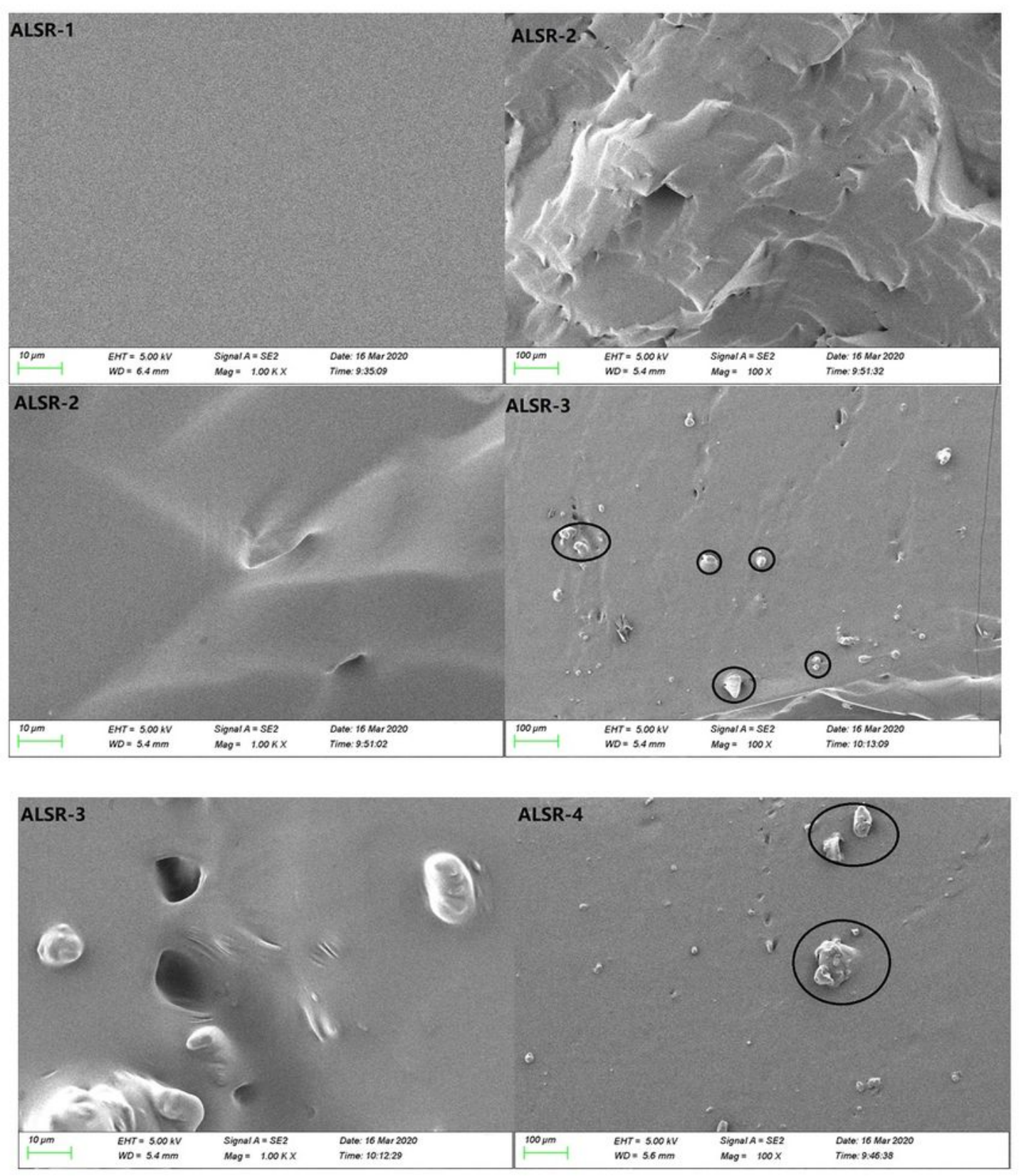

\section{Figure 3}

Cross-sectional SEM images of (ALSR-1) neat ALSR, (ALSR-2) SEUG/ALSR composite rubber with 5 wt\% SEUG content, (ALSR-3) SEUG/ALSR composite rubber with 10 wt\% SEUG content, and (ALSR-4) SEUG/ALSR composite rubber with 15 wt\% SEUG content. 


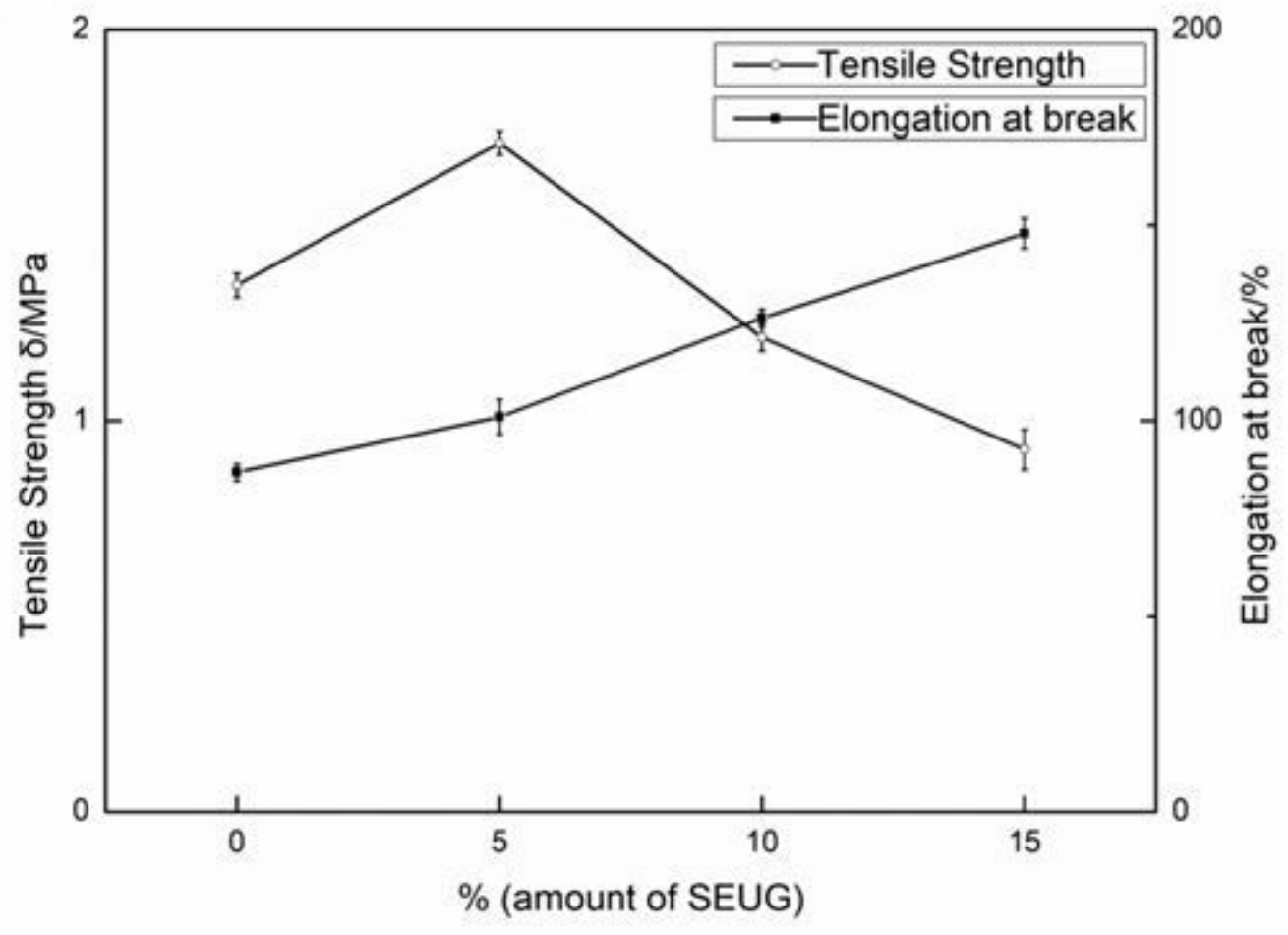

Figure 4

Tensile strength and elongation at break of SEUG/ALSR composite rubbers with different contents of SEUG. 


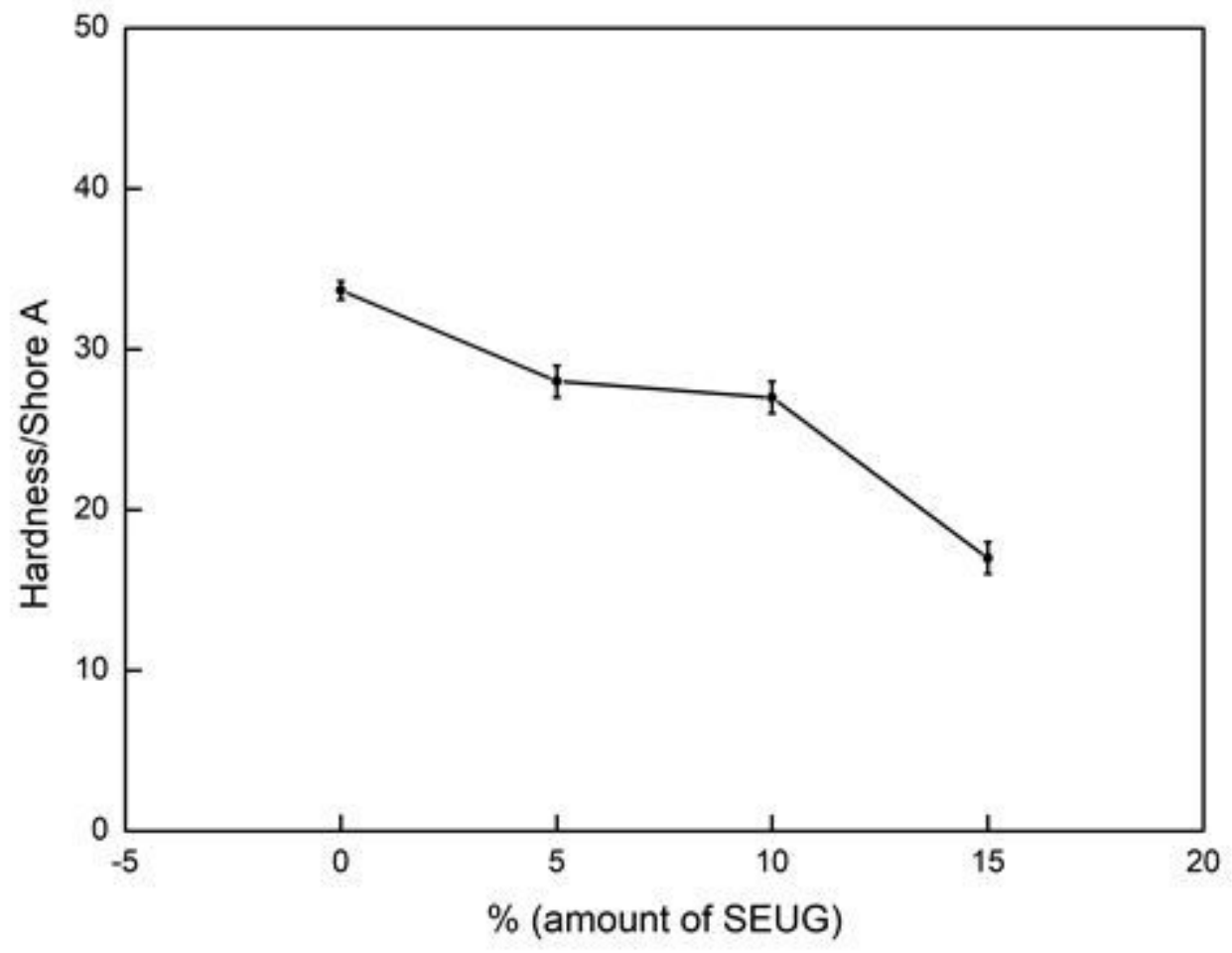

Figure 5

Shore A hardness values of the SEUG/ALSR composite rubbers with different contents of SEUG.

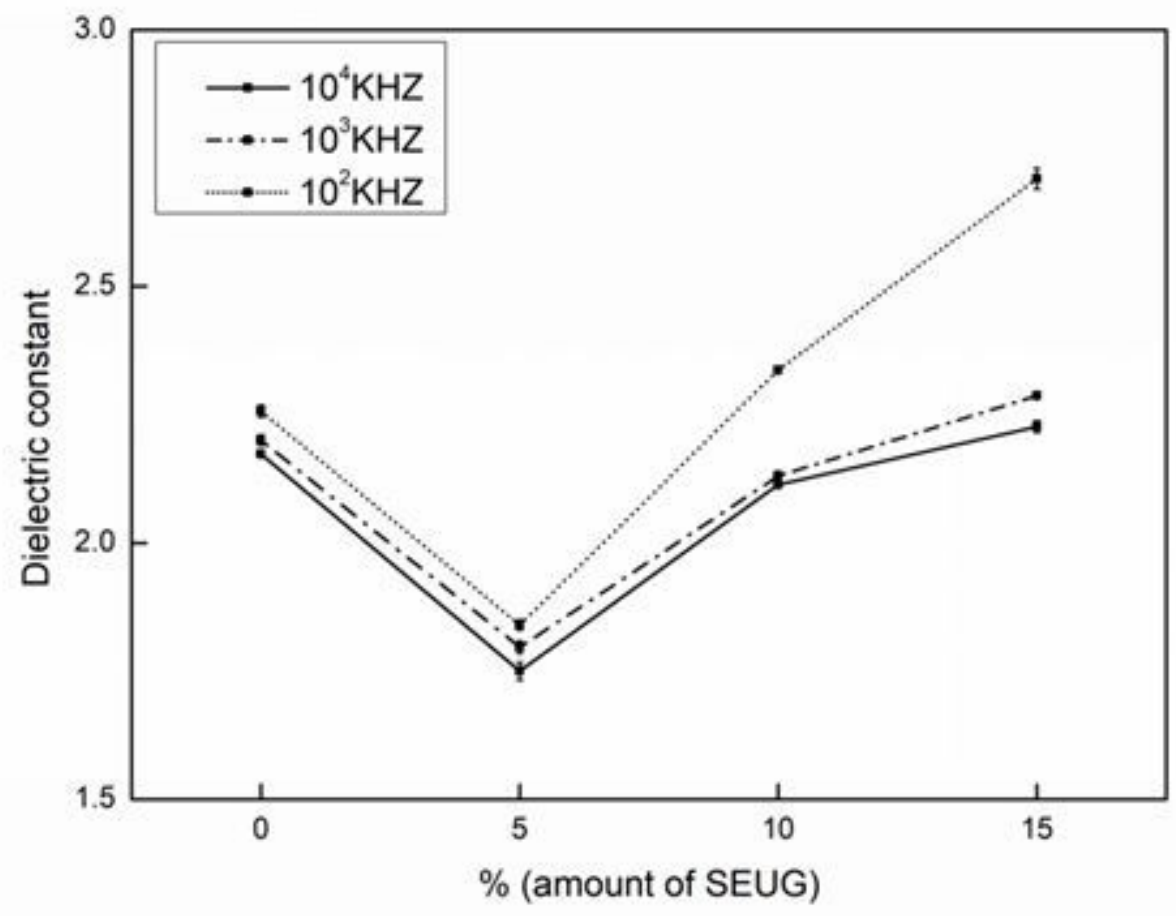


Figure 6

Dielectric constants of the SEUG/ALSR composite rubbers with different contents of SEUG.

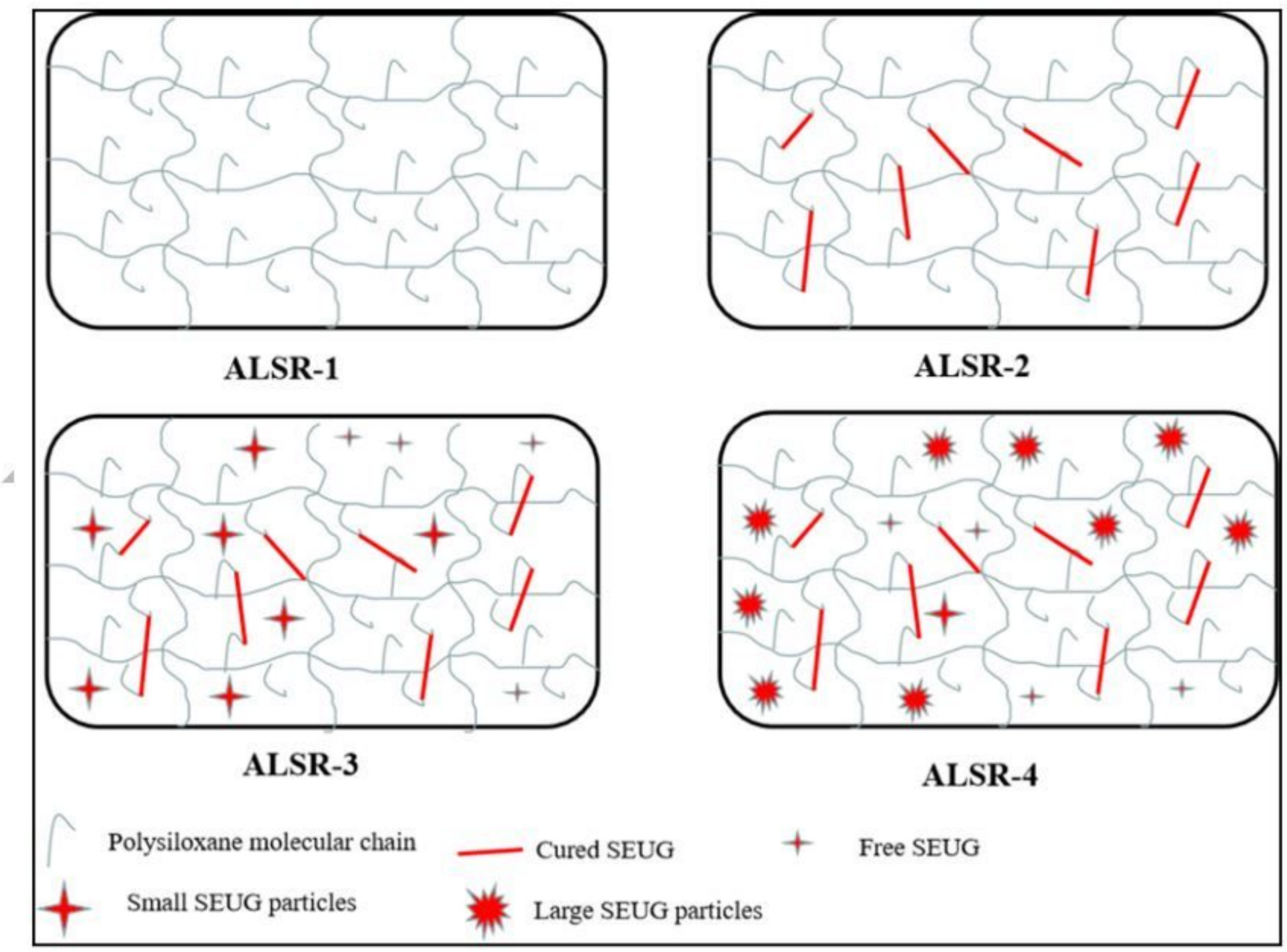

Figure 7

Possible mechanism through which the SEUG affected the low-dielectric properties of the ALSR. 


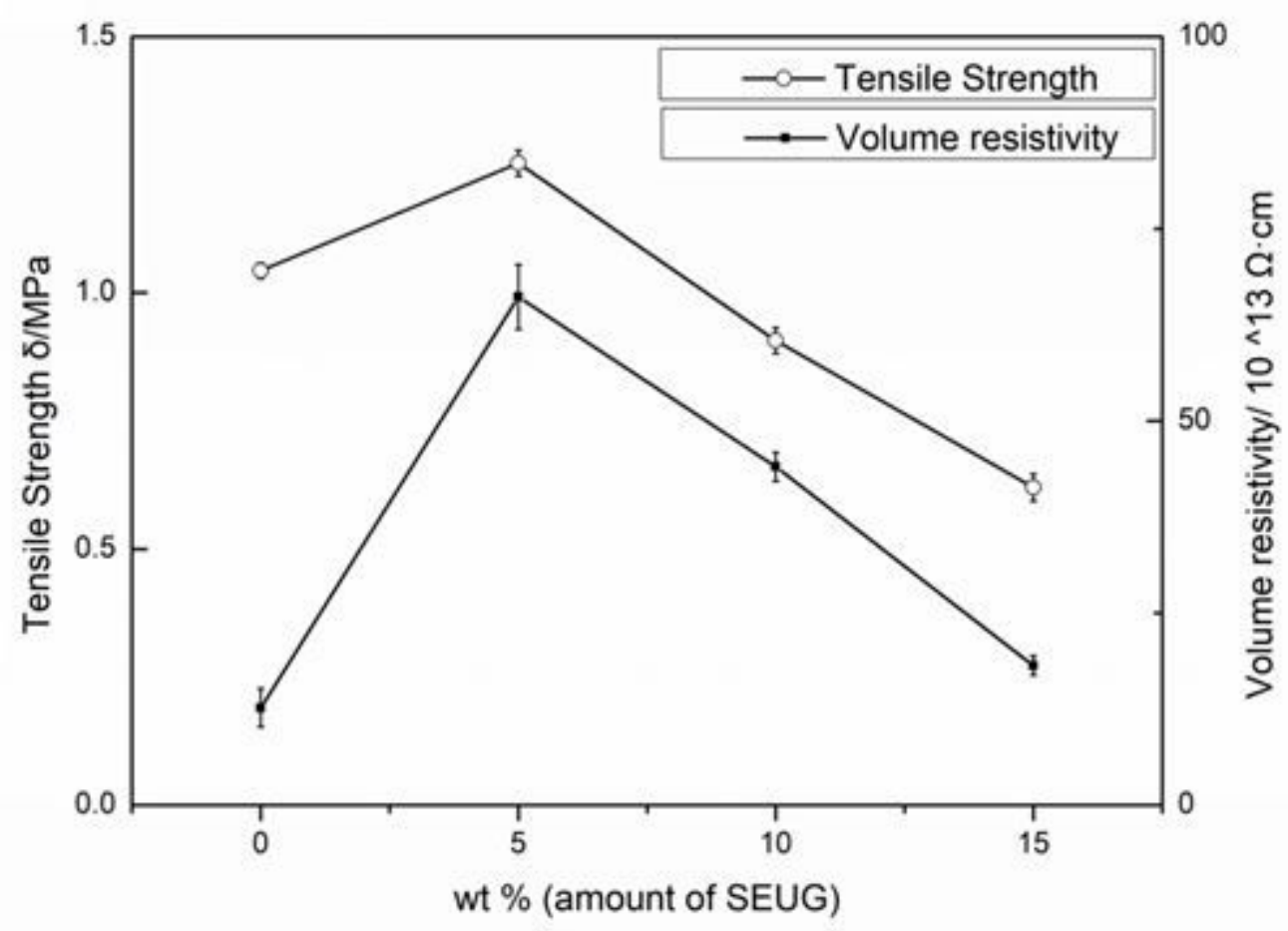

Figure 8

Tensile strength and volume resistivity of the SEUG/ALSR composite rubbers after $\mathrm{HCl}$ resistance tests.

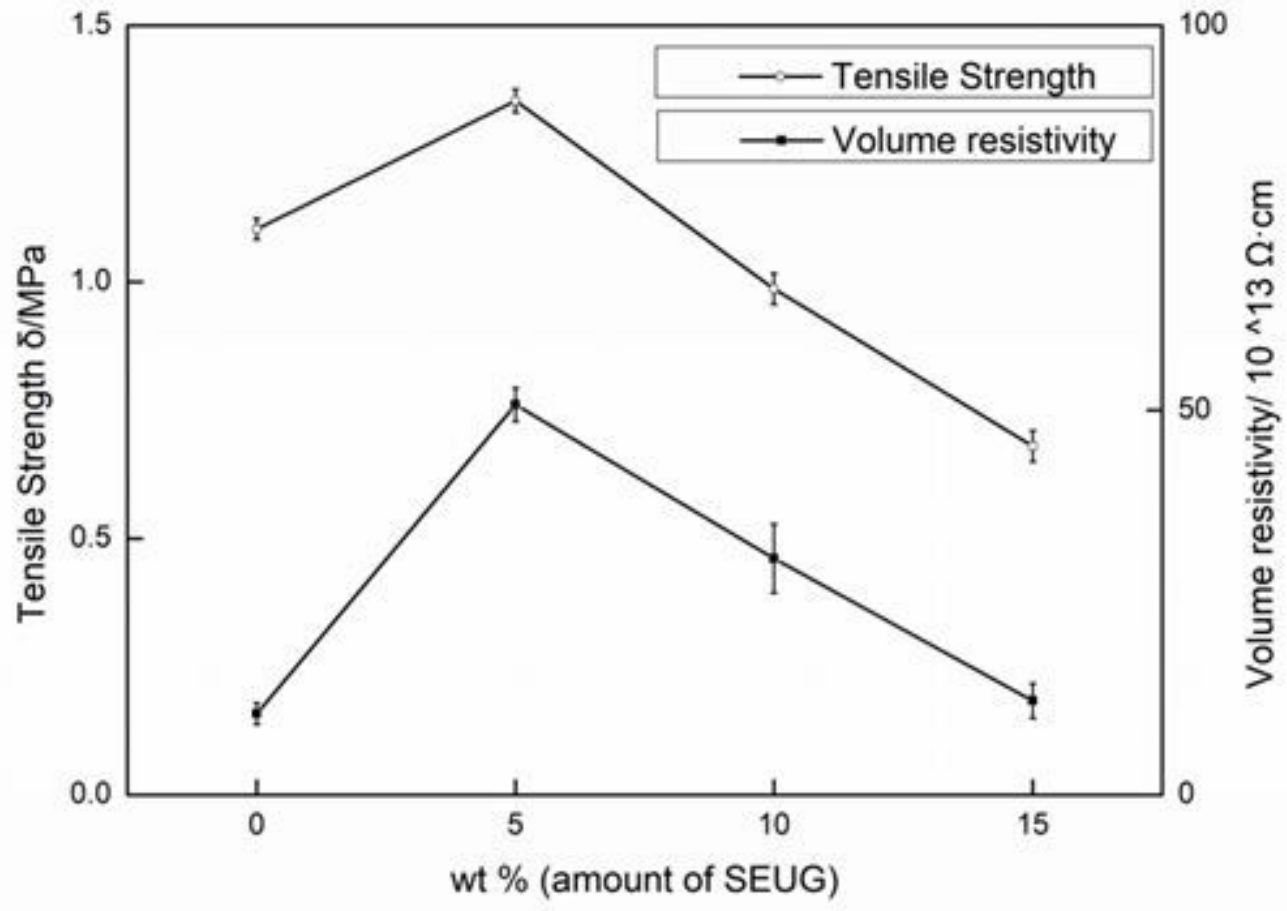

Figure 9 
Tensile strength and volume resistivity of the SEUG/ALSR composite rubbers after $\mathrm{NaCl}$ resistance tests.

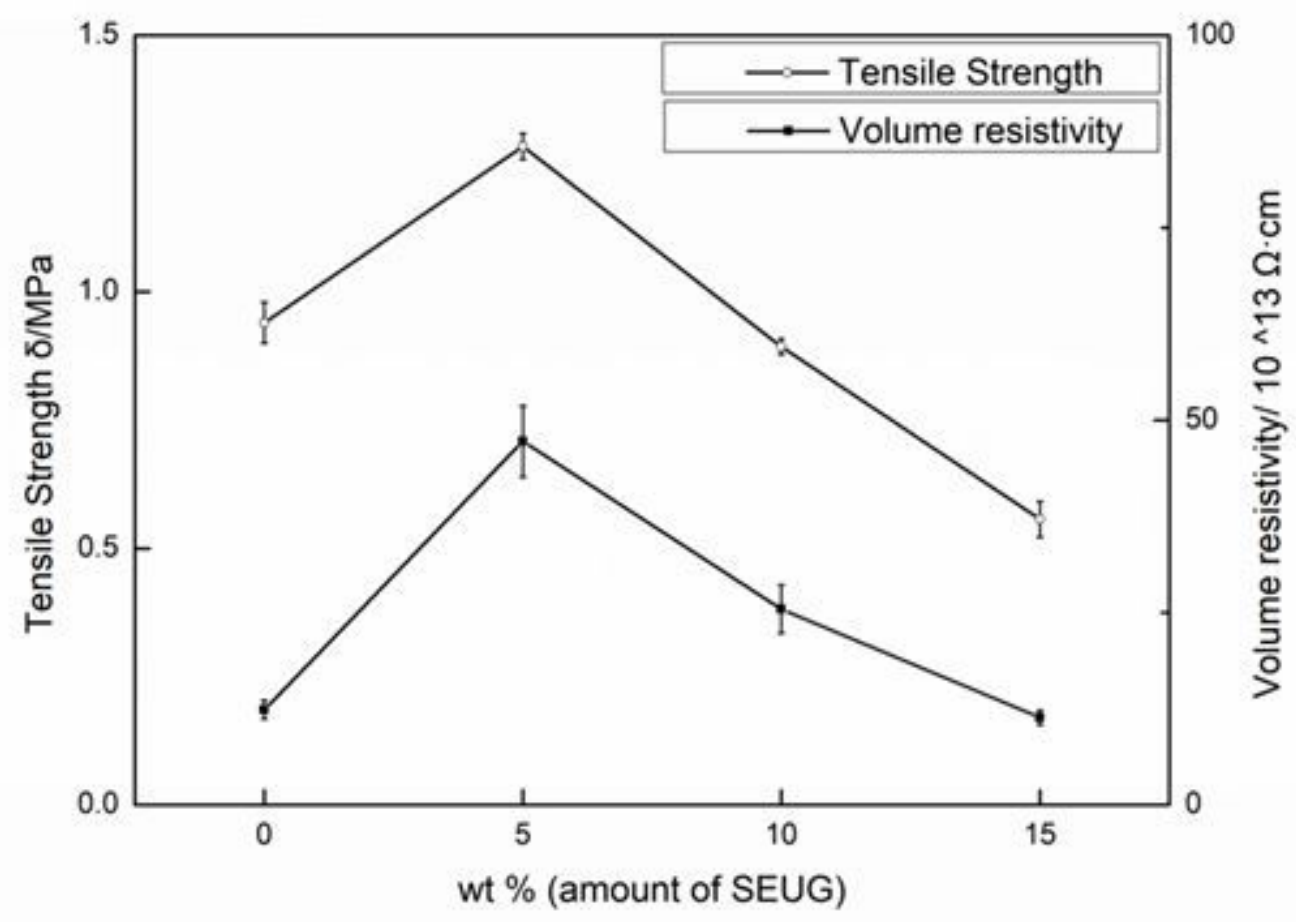

Figure 10

Tensile strength and volume resistivityof the SEUG/ALSR composite rubbers after oil resistance tests.

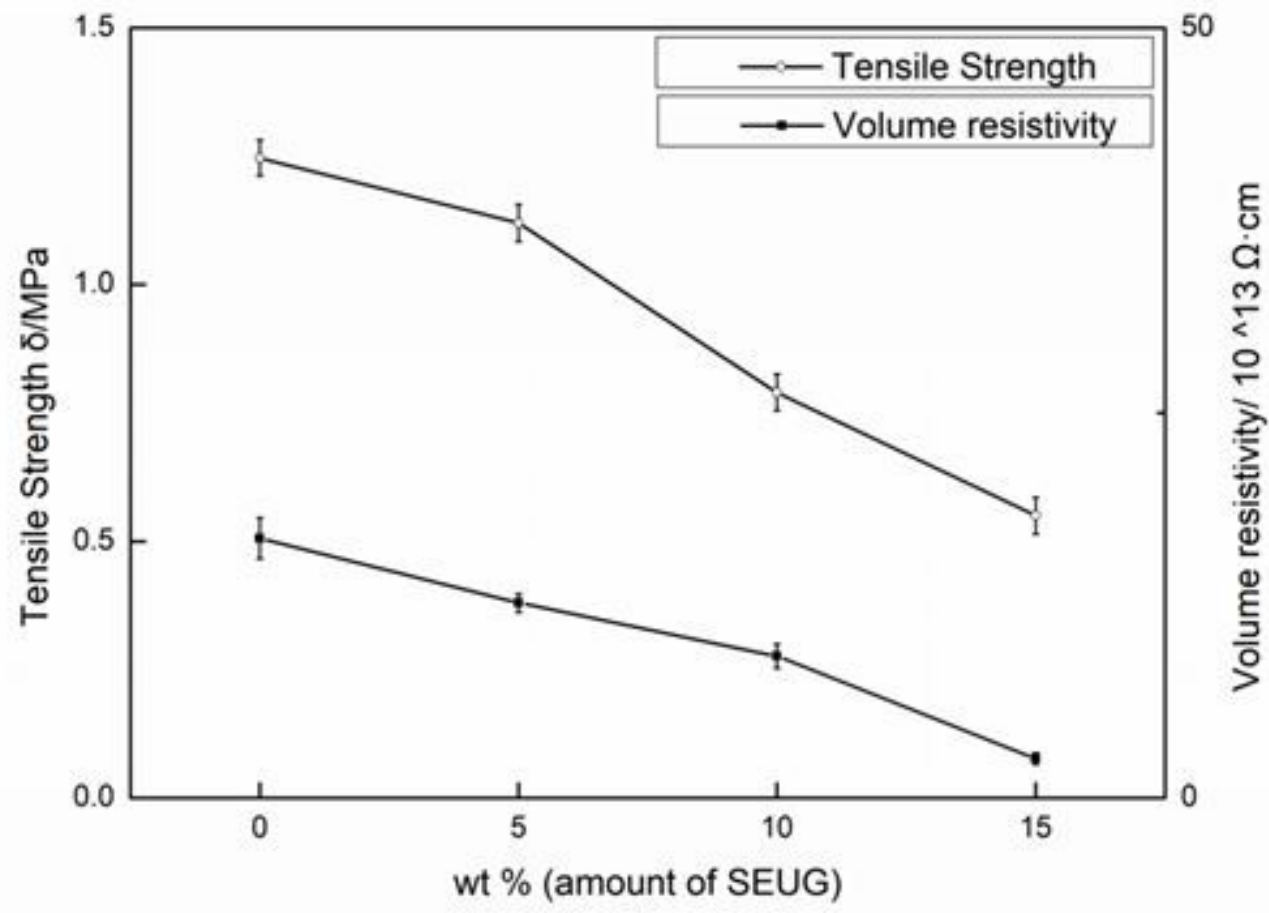

Figure 11 
Tensile strength and volume resistivity of the SEUG/ALSR composite rubbers after $\mathrm{NaOH}$ resistance tests.

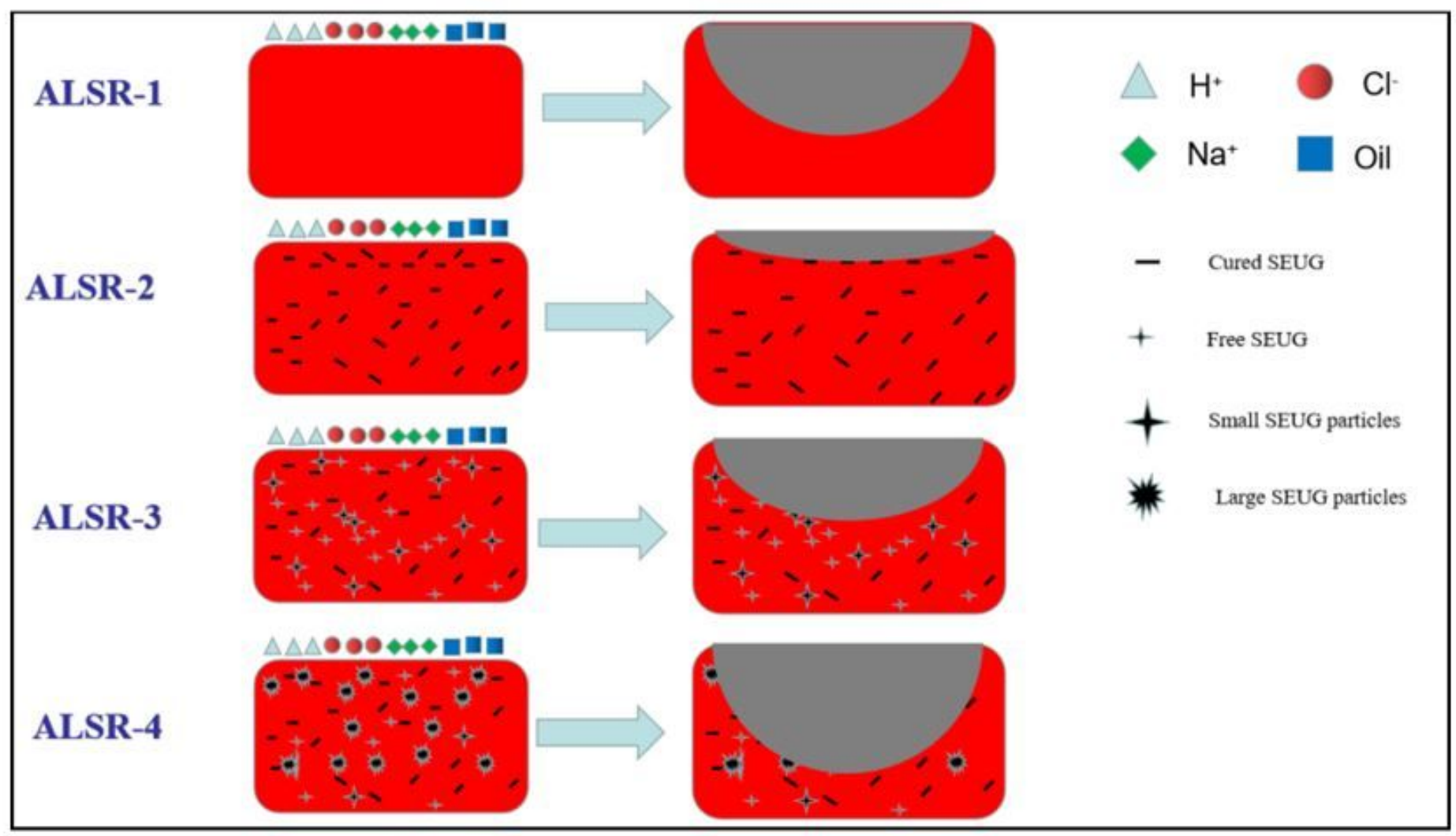

Figure 12

Possible mechanism through which the SEUG enhanced the $\mathrm{HCl}, \mathrm{NaCl}$, and oil resistances of the ALSR.

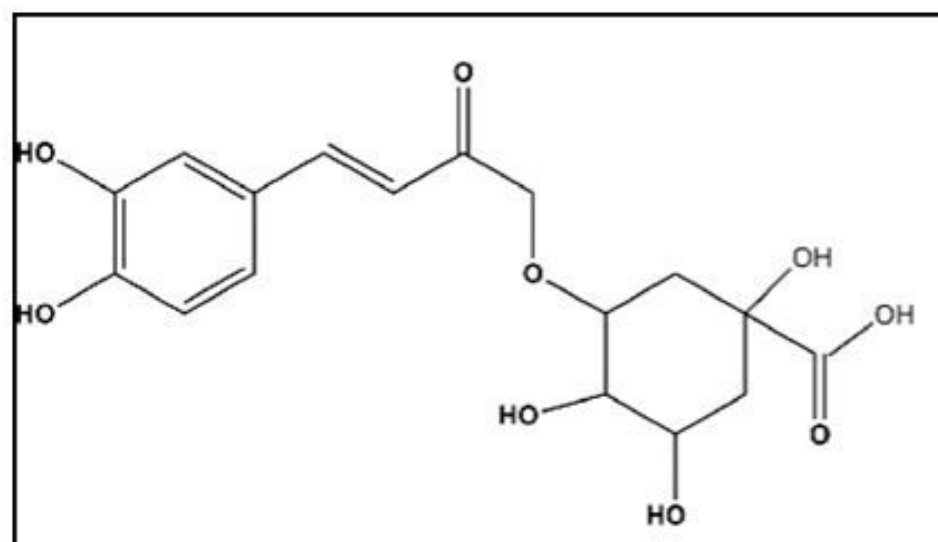

Chlorogenic acid

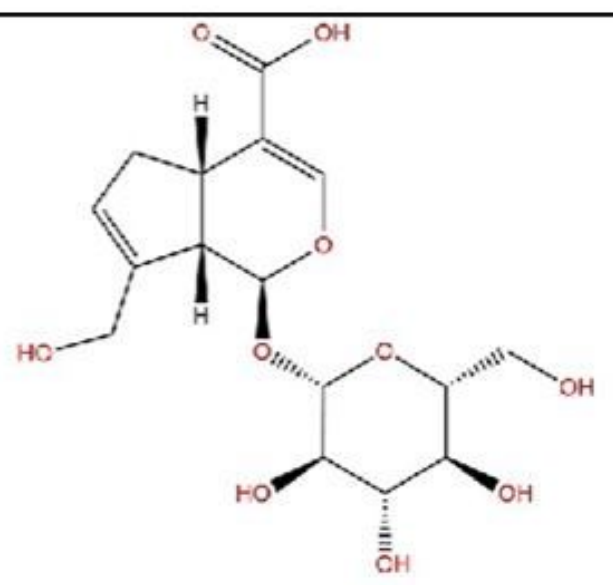

Geniposide crystal

\section{Figure 13}

Structures of bio-based macromolecules remaining in the EUG. 


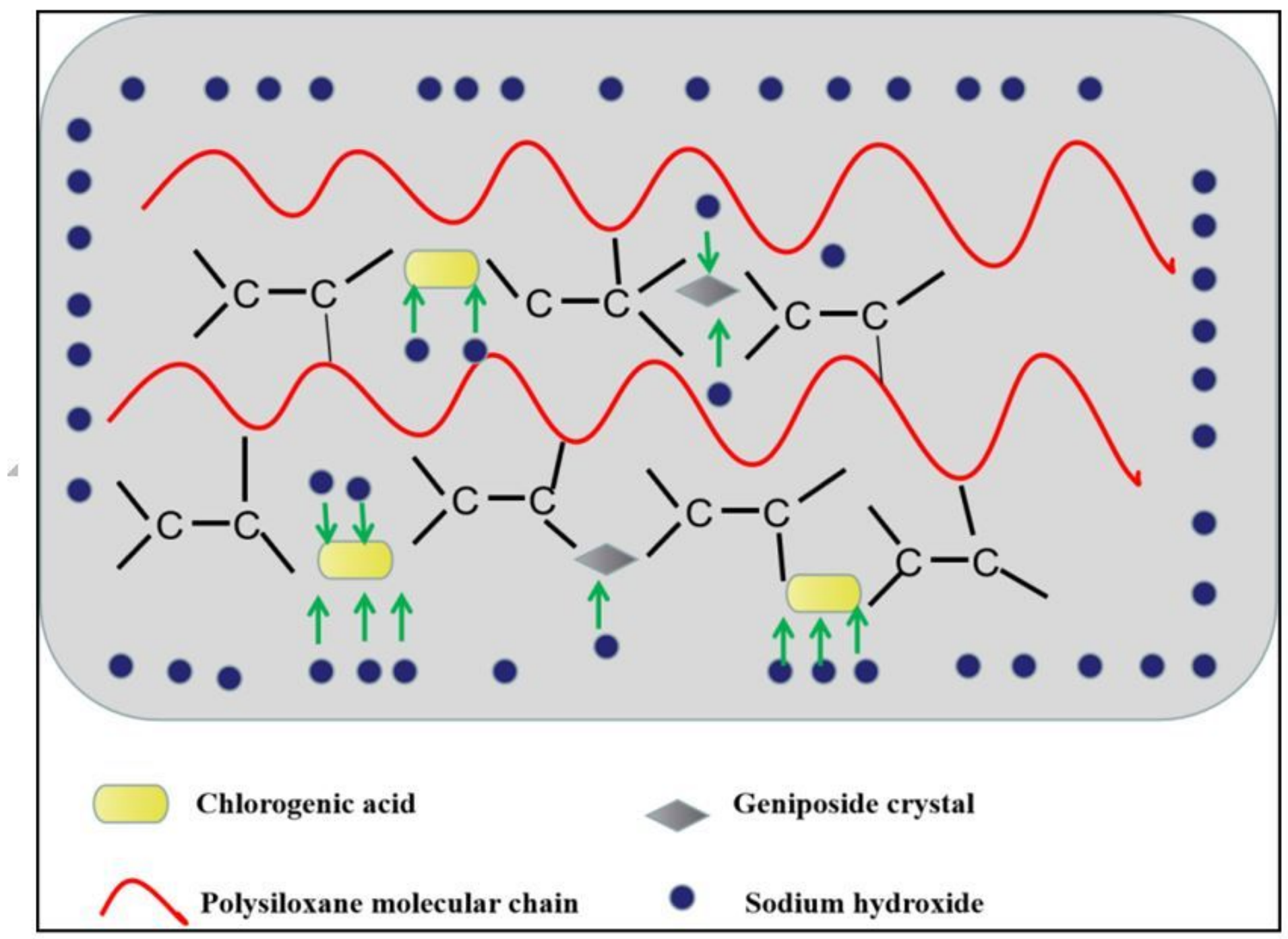

Figure 14

Possible mechanism through which the SEUG reduced the $\mathrm{NaOH}$ resistance of the ALSR.

\section{Supplementary Files}

This is a list of supplementary files associated with this preprint. Click to download.

- Scheme1.jpg 